Psychological Medicine, 1990, 20, 243-248

Printed in Great Britain

\title{
EDITORIAL
}

\section{The semantic problems of psychiatry ${ }^{1}$}

Medical problems are commonly discussed in terms of 'diseases'; and although important decisions may be made, especially in emergency situations, without the use of this terminology, it is unlikely that a medical language that dispenses with it will be elaborated. Names of diseases have widely varying factual implications; i.e. the types of observation that are required to test diagnostic statements that use them vary widely. If they are to be used in statements purporting to be scientific, it is essential that these varying implications should be made explicit. Analysis of medical usages of the names of diseases, both in my own speciality of respiratory disease (Scadding, 1959, 1963, 1981) and more generally (Scadding, 1967, 1972, 1975; Campbell et al. 1979), has led me to propose the following statement which aims to do this.

In medical discourse, the name of a disease refers to the sum of the abnormal phenomena displayed by a group of living organisms in association with a specified common characteristic, or set of characteristics, by which they differ from the norm for their species in such a way as to place them at a biological disadvantage.

This statement does not aspire to be a definition of 'disease-in-general', which Taylor (1980) and Häfner (1987), among others, have sought. Popper (1945) pointed out many years ago that 'essentialist' definitions of this sort, attempting the impossible task of revealing the essence of the definiendum, have no place in science; my statement is methodologically nominalist, with the limited objective of making explicit the factual implications of current medical usages of the names of diseases, accepting their logical heterogeneity. I have recently outlined some of the consequences of acceptance of this, which I shall call 'the general statement' (Scadding, 1988). Here, I propose to consider its possible relevance to problems of nosology and classification in psychiatry of which even outsiders to this speciality must be aware, and of which my awareness has been sharpened by two previous invitations to contribute to this journal (Scadding, 1980, 1982).

\section{'CLASSIFICATION' OF DISEASES}

The general statement is primarily concerned with the clarification of medical nomenclature; but it has important consequences for classification, since it indicates the impossibility of any attempt to classify diseases in a comprehensive hierarchical system. In the 17th century, Sydenham (1696) suggested that 'all Diseases should be reduced to certain and definite Species, with the same diligence we see it is done by Botanick writers in their Herbals'. This suggestion was feasible because at that time diseases were definable only by clinical description and (presumably) were regarded as causes of the symptoms and signs observed in patients. Today, we must acknowledge that the name of a disease may refer to no more than a consistent syndrome whose cause is not known or various, but whose recognition is useful because study of previous cases permits prognosis, and may have discovered helpful therapeutic procedures. It may refer to the effects of a specified disorder of structure or function, correction or amelioration of which may be possible, even though its cause remains unknown. It may refer to the effects of a specified causal agent or process (aetiology). Thus, the causal implications of a diagnosis in the current disease terminology vary greatly. This heterogeneity must be openly acknowledged; if it is, the names of diseases can be used in concise diagnostic statements which take causal explanation only as far as the available evidence allows, and do not convey unjustified implications. As I have commented elsewhere (Scadding, 1980), attempts to elaborate a unified concept of disease are doomed to failure, and can lead only to corfusion.

Thus, current nosology deals with a list of diseases defined by different sorts of criteria. Among

'Address for correspondence: Professor J. G. Scadding, 18 Seagrave Road, Beaconsfield, Bucks, HP9 ISU. 
those defined by criteria of one sort - e.g. infectious diseases - it is possible to define mutually exclusive categories; but categories defined by criteria of different sorts cannot be expected a priori to be mutually exclusive. Only if it were possible to order the defining criteria hierarchically would a logically rigorous classification of diseases be possible. Such a classification with aetiology as the primary criterion is an attractive idea, but is not feasible. Limitations of knowledge would require an enormous residual category 'of unknown aetiology' in such a classification, and this would contain many cases in which the involvement of a possible causal factor could neither be asserted nor confidently denied. And even though when aetiology is known it is usually adopted as the primary defining characteristic, a disorder of structure or function sometimes has such important practical implications that it takes priority in diagnostic categorization.

\section{COMPOUND DIAGNOSTIC CATEGORIES}

Although a hierarchical classification of diseases is not possible, useful diagnostic categories may be defined by criteria from more than one field of study. Definition in this way constitutes a compound diagnostic category. Many apparently simple diagnostic terms refer to compound diagnostic categories: e.g. general paralysis of the insane (dementia paralytica) is defined in three fields; syndrome, neuropathology, and aetiology. This device permits an advance in knowledge to be incorporated into terminology without a claim to universality, avoiding the absurdities of universal theories of disease. If a factor $F$ is found to be causally related to the syndromally-defined disease $K$, the compound diagnosis $K(F)$ can be made without implying that all $K$ is due to $F$ or that $K$ is the only ill-effect of $F$. If $F$ is found to be of sufficient importance, it may well become the basis for definition of a primary diagnostic category, which, though it may include some cases not conforming to the syndrome $K$, and not include all those that do, may come to replace it in common usage. It must be recognized that when it becomes possible to redefine a syndromally-defined disease in any other way, the group of patients study of whom provides the description of the disease under the new definition is unlikely to be identical with that which provided it under the old. Thus, the syndromally defined category 'phthisis' was correctly associated first with the morbid-anatomical change tuberculosis, and then with causation by the tubercle bacillus, leading to the current name 'pulmonary tuberculosis'; this is a compound category, with anatomical and aetiological terms. It is certain that some patients given the diagnosis of phthisis before Koch's discovery of the tubercle bacillus were not suffering from tuberculosis; and the current category 'tuberculosis' includes cases that would not have been included in any of the syndromally defined categories which preceded it in nosology.

This seems to me relevant to discussion of aetiology in psychiatry. For instance, if a causal factor $X$, such as a chromosomal abnormality, a biochemical defect or a viral infection is found and validated for schizophrenia, it is not to be expected that the disease defined as that caused by $X$ will be identical with schizophrenia as currently defined in descriptive terms. The adoption of a compound diagnostic category, schizophrenia $(X)$, will permit reference to those cases associated with this factor, without implying that all schizophrenia is due to $X$. If it appears that $X$ is important in decisions about treatment, prevention or prognosis, it may become the defining characteristic for a new primary diagnostic category, taking precedence over schizophrenia for those cases in which it is concerned.

\section{IS THIS A DISEASE?}

The phrase 'at a biological disadvantage' in the general statement specifies briefly the sort of considerations that lead to a deviation from norm being regarded as indicative of disease. For many diseases, especially those definable aetiologically or in terms of disorders of structure or function, application of this criterion is not difficult, since disability and risk to life are obvious. If they are not obvious, 'disadvantage' is to be assessed in relation to the group of all those with the relevant characteristic, rather than the individual; arguments about whether a defined group should be said to be suffering from a disease, or about the magnitude of deviations from norm of function or 
structure that will be accepted as indicative of disease, are to be decided by criteria based on statistical studies of relevant populations, the results of which are open to general inspection.

'Biological disadvantage' thus provides a criterion which defines areas of doubt and indicates how they can be reduced in size. The question arises: does 'biology', in this context, include all the life sciences? This is considered below. Here I will note that in medical discourse acceptance of a syndrome as a disease seems to imply a belief that further study may discover an underlying disorder of structure or function and/or a causal factor or process, and thus permit redefinition in terms with more objectively demonstrable causal implications than clinical description.

This analysis not only clarifies medical usages of the names of disease, but also conforms in a general way with lay usage. Non-medical people tend to regard fewer conditions for which they may seek advice as diseases than the doctors they consult (Campbell et al. 1979), largely because they think of a disease as something serious, which may lead to disability or threaten life.

\section{THE CONCEPT OF 'DISEASE' IN PSYCHIATRY}

Kendell (1975) has drawn attention to the difficulty of applying the criterion of biological disadvantage in psychiatry, most of whose diagnostic categories are syndromally defined. Is it proper to regard them as diseases? Sometimes an attempt is made to side-step this question by avoiding the word 'disease' and using instead such terms as syndrome, disorder or illness. This contributes little to clarity unless the intended implications of these terms are made explicit, as the general statement seeks to do for the names of diseases.

For instance, although DSM-III-R (American Psychiatric Association, 1987) recognizes that some mental disorders can be attributed to organic diseases, it avoids the use of the word 'disease' even for these, recording them on a separate axis as Physical Disorders. Thus 'disorder' seems to be a permitted equivalent for the forbidden word 'disease'. As a definition of its subject matter, Mental Disorders, DSM-III-R offers the statement that each 'is conceptualised as a clinically significant behavioral or psychological syndrome or pattern' which is associated with undesirable features of various sorts, and is 'currently considered a manifestation of a behavioral, psychological, or biological dysfunction in the person'. This statement leaves so many questions open that it can hardly be called a definition. Several points in it call for comment.

The distinction between biological, psychological and behavioural, as used in this context, requires examination. Since psychology and behavioural science are aspects of the study of living organisms, they are subsumed in biology, in its widest sense; but I will take it that 'biology' is being used here to mean the study of living organisms directed towards explanation in physico-chemical terms, and when I use this word without qualification, it will be in this limited sense. Some disorders of behaviour can already be explained in this way; and it is to be expected that with advances in knowledge, more and more of psychology and behavioural science will become biological. In the meantime, of course, these disciplines advance by methods other than those of chemistry and physics, and offer explanations that do not yet reach this level.

The DSM-III-R statement mentions psychological and biological dysfunctions disjunctively, without discussion of the relevance of differences between them to nosology. I suggest that the most important difference is in the objectivity with which dysfunctions currently described in these ways can be assessed. For biological function there exists a generally agreed corpus of knowledge of mechanisms and norms, so that the hypothesis that symptoms are due to a biological dysfunction can be tested objectively, without the need for subjective judgements. It is presumably because of difficulties in dealing similarly with patterns and syndromes not currently attributable to biological dysfunction that the DSM-III-R statement admits the possibility of such judgements. 'Conceptualised', 'clinically significant', and 'considered' leave decision in doubtful cases dependent upon the subjective judgment of unspecified experts, presumably psychiatrists. Who decides that a behavioural pattern is clinically significant, and to be explained as a manifestation of a behavioural dysfunction? What are the practical implications of this attribution, apart from those that follow from failure to find a biological explanation?

In 1945, Popper observed that 'methodological nominalism is generally accepted in the natural 
sciences. The problems of the social sciences, on the other hand, are still for the most part treated by essentialist methods'. This observation remains apposite: progress in the social sciences is to be judged by the extent to which it becomes possible to apply methodological nominalism to them, bringing them into the ambit of biology, and increasing the area within which hypotheses can be tested by objective criteria.

\section{THE FALLACY OF REIFYING DISEASES AS CAUSES}

The general statement makes manifest the varying causal implications of diagnostic statements in a disease terminology. Uncritical reification of diseases as causes is implicit in colloquial usage, and constant vigilance is required to exclude it from medical discourse. For instance, the text of DSMIII-R sometimes suggests that Disorders and Syndromes are causes of symptoms: e.g. 'The diagnosis (of Schizophrenia) is not made if the symptoms are due to a Mood Disorder or Schizoaffective Disorder', though elsewhere this pitfall is avoided by a more judicious wording, such as 'not part of' or 'does not meet the criteria for'. This suggestion is reinforced by the use of initial capital letters for their names; and also by the use of the word 'specific', without clarification of its implications, in the reference to diagnosis as 'the process of identifying specific mental or physical disorders'. This point has been noted by others (Boyd et al. 1984; Edlund, 1986).

In dealing with groups of patients whose condition cannot be related to specifiable physical disorders or causal agents, it is especially important to avoid the danger of reifying abstract concepts, whether they be called diseases, disorders, syndromes or anything else, as causes; and in such contexts it is arguable that these concepts are a hindrance to clear communication. The study of the classification of mental disorder in primary care by Jenkins et al. (1988) provides an example of this. This study was designed to test how consistently two classification systems can be applied by general practitioners to the categorization of patients of a type which they must see frequently, those with depression or anxiety, possibly accompanying established organic disease. Left to express their conclusions in their own words, participants generally gave descriptive statements which incorporated their assessments of psychological, personality and social features, about which they agreed fairly well; but the concordance of their responses to the request for 'diagnoses' of mental disorder in terms of two formal classification systems was poor. It seems that faced with a patient in whom mood changes accompanied various social and economic stresses and recognized physical diseases, they preferred to describe the situation in informal terms, rather than commit themselves to a formal diagnosis which would imply that the changed mood should be regarded as due to a postulated 'mental disorder', for which a biological basis may be implied though none has been established, rather than as a response to evident stresses, not unexpected in the light of their assessment of the patient's personality. After participants had made their initial formulations, they were informed of events over the next two years in each patient's case, and asked to review their diagnoses. Since no patient had deteriorated to any great degree, the question whether diagnostic categorization of more severe mental disorders would be more consistent could not be studied. Perhaps the diagnostic terms of the formal classifications would have been applied to these more often and more concordantly, since common sense suggests that it is in the more severe and persistent affective disorders that biological factors are most likely to outweigh environmental and social stresses in importance as causal factors.

This dilemma is reflected in the variety of adjectives which have been used to qualify 'depression' when used as a diagnostic term, informatively reviewed by Lewis (1971). Most of these seem to be concerned with distinction between cases in which there are, and are not, thought to be grounds for supposing that the symptoms are more severe than can be accepted as an expected reaction to known stresses. 


\section{SZASZ AND THE MYTH OF MENTAL ILLNESS}

The responses of the general practitioners in this study reflected their recognition that there is a grey area within which they were not prepared either to assert or categorically to deny the possibility that biological factors might be important in the causation of abnormalities of mood and behaviour.

This sceptical approach contrasts with the dogmatic iconoclasm of Szasz (1961), who seeks to discredit any biological explanation for syndromes of abnormal affect and behaviour. In his enthusiasm, he sometimes shows little understanding of aspects of 'organic' medicine relevant to his theme. For instance, in a paper attacking the concept of schizophrenia as a disease (Szasz, 1976), he suggests that dementia paralytica was accepted as a disease only after it was found "that the brain tissue of paretics, and of paretics only, harboured hordes of Treponema pallida'; whereas the relationship of the symptom-complex of this disease to a late stage of syphilis was widely thought probable before it was objectively demonstrated, and in this manifestation of neurosyphilis, organisms are not found in all cases and when found are not numerous, so that in many cases the association with syphilis remains inferential. And in the same paper, he makes the astonishing statement that 'until recent times, physicians spoke of venereal diseases collectively: genuine classification of these diseases occurred only after discoveries in microbiology provided the necessary tools for it'; whereas the pox and the clap were defined by clinical description and clearly distinguished from each other long before Pasteur initiated bacteriology. Nevertheless, these blind spots do not invalidate his protest against the undesirable consequences of the reification of Mental Disorders as causes, and against poor definition of the proper boundaries of medical responsibility.

\section{'BIOLOGICAL DISADVANTAGE' AS A DEMARCATION CRITERION}

The problem to which Szasz's polemics draw attention is really that of the demarcation of medical responsibility. I suggest that 'biological disadvantage' is a useful criterion here. If this criterion evidently applies, there should be no doubt about the propriety of medical intervention, especially when there is a defect of function or structure which can be alleviated or an causal agent which can be removed or neutralized. If it evidently does not, the problem is unequivocally not a medical one. Behaviour that deviates from politically accepted norms, with no suggestion that it originates in an abnormality causing biological disadvantage, is obviously not a medical concern. And it is evidently important that doctors should recognize changes in mood and behaviour that can be accepted as expected reactions to life events, but their specifically medical responsibility in helping those affected in this way is limited to initial diagnosis and possible short-term pharmacological help. But there is a grey area, illustrated by the study of Jenkins et al. (1988), where it is not surprising that doctors are divided in their opinions about whether subjective distress and/or behavioural changes should be regarded as indicative of disease, and therefore a proper subject for medical treatment. If the methodological approach summarized in the general statement is adopted, and doubt is openly admitted, this difference of opinion should not adversely affect the individual patient. Whether the doctor simply records that the patient is depressed, or makes a diagnosis of Depression, he or she will presumably enquire about evident social and personal stresses and refer the patient to appropriate agencies to help in coping with them, and consider whether pharmacological intervention is advisable. For the advancement of medical science, the delineation of syndromes, without prior commitment to views on causation, is a well-tried starting-point for studies to elucidate pathenogenesis and aetiology, and extend the area within which practice can be based upon objective biological knowledge.

J. G. SCADDING

\section{REFERENCES}

American Psychiatric Association (1987). Diagnostic and Statistical Manual of Mental Disorders, 3rd edn, revised. American Psychiatric Association: Washington, D.C.
Boyd, J. H., Burke, J. D., Gruenberg, E., Holzer, C. E., Rae, D. S., George, L. K., Karno, M., Stoltzman, R., McEvoy, L. \& Nestadt, G. (1984). Exclusion criteria of DSM-IIl : a study of co-occurrence 
of hierarchy-free syndromes. Archives of General Psychiatry 41, 983-989.

Campbell, E. J. M., Scadding, J. G. \& Roberts, R. S. (1979). The concept of disease. British Medical Journal ii, 757-762.

Edlund, M. J. (1986). Causal models in psychiatric research. British Journal of Psychiatry 148, 713-717.

Häfner, H. (1987). The concept of disease in psychiatry. Psychological Medicine 17, 11-14.

Jenkins, R., Smeeton, N. \& Shepherd, M. (1988). Classification of mental disorder in primary care. Psychological Medicine, Monograph Supplement 12.

Kendell, R. E. (1975). The Role of Diagnosis in Psychiatry. Blackwell: Oxford.

Lewis, A. (1971). 'Endogenous' and 'exogenous': a useful dichotomy? Psychological Medicine 1, 191-196.

Popper, K. R. (1945). The Open Society and its Enemies. Kegan Paul: London.

Scadding, J. G. (1959). Principles of definition in medicine. Lancet i, 323-325.

Scadding, J. G. (1963). Meaning of diagnostic terms in bronchopulmonary disease. British Medical Journal ii, 1425-1430.

Scadding, J. G. (1967). Diagnosis: the clinician and the computer. Lancet ii, 877-882.

Scadding, J. G. (1972). The semantics of medical diagnosis. Biomedical Computing 3, 83-90.
Scadding, J. G. (1975). The classification of disease. In Advances in Medical Computing (ed. J. Rose and J. H. Mitchell), pp. 5-9. Churchill Livingstone: Edinburgh.

Scadding, J. G. (1980). The concepts of disease : a reply. Psychological Medicine 10, 425-427.

Scadding, J. G. (1981). Talking clearly about broncho-pulmonary disease. In Scientific Foundations of Respiratory Medicine (ed. J. G. Scadding and G. Cummings), pp. 727-734. Heinemann: London. Scadding, J. G. (1982). Review of What is a case? (ed. J. K. Wing, P. Bebbington and L. H. Robbins). London: Grant McIntyre, 1981. Psychological Medicine 12, 207-208.

Scadding, J. G. (1988). Health and disease: can medicine help philosophy? Journal of Medical Ethics 14, 118-124.

Sydenham, T. (1696). The Whole Works of that Excellent Physician Dr Thomas Sydenham, translated by John Pechy, M.D. Richard Wellington and Edward Castle: London.

Szasz, T. (1961). The Myth of Mental Illness. Harper and Row: New York.

Szasz, T. (1976). Schizophrenia: the sacred symbol of psychiatry. British Journal of Psychiatry 129, 308-316.

Taylor, F. K. (1980). The concepts of disease. Psychological Medicine $10,417-426$. 